\title{
Análisis del resultado de pruebas hepáticas en pacientes psoriáticos tratados con metotrexato: Estudio retrospectivo
}

\author{
Hernán Correa $\mathbf{~}^{1}$, N atalia Paredes $\mathrm{S}^{2}$. \\ Serum liver tests in patients \\ treated with methotrexate. \\ $A$ retrospective analysis
}

Background: Methotrexate is one of the best systemic treatments for psoriasis. However it has significant adverse effects such as myelotoxicity and hepatotoxicity. Aim: To evaluate serum liver test in psoriatic patients treated with methotrexate. Material and Methods: Retrospective review of medical records of psoriatic patients treated with methotrexate between the years 2000 and 2005. All patients received a minimum of $7.5 \mathrm{mg}$ weekly of methotrexate, for at least 4 weeks. Results: Sixty three patients were included. Mean cumulative dose of methotrexate was $576 \mathrm{mg}$. Thirty two percent had alterations in liver tests, but only $9 \%$ had values that duplicated the upper limit of normal range of aminotransferases or alkaline phosphatases or a serum bilirubin over $2 \mathrm{mg} / \mathrm{dl}$. We did not find a direct relationship between the dose of methotrexate and the magnitude of liver test alterations. Only one patient exceeded $1.5 \mathrm{~g}$ as cumulative dose. A liver biopsy performed to him, did not show signs of fibrosis. Conclusions: This retrospective study does not show a direct relationship between weekly doses, cumulated dose and length of treatment with methotrexate, and the degree of alteration of serum liver tests (Rev Méd Chile 2007; 135: 1002-8).

(Key words: Liver function tests; Methotrexate; Transaminases)

Recibido el 20 de julio, 2006. Aceptado el 6 de marzo, 2007.

${ }^{1}$ Servicio de Dermatología, Hospital Sótero del Río. ${ }^{2}$ Escuela de Medicina, Pontificia Universidad Católica de Chile, Santiago de Chile.

L a psoriasis es una enfermedad crónica de la piel que afecta aproximadamente $2 \%$ a $3 \%$ de la población mundial ${ }^{1,2}, 25 \%$ de los pacientes presentan una psoriasis moderada a severa, que afecta la calidad de vida, requiriendo en algún

Correspondencia a: Dr. Hernán Correa G. Las Urbinas 81 Depto. 2-A Providencia. Santiago de Chile. Fax 2338304. E mail: hcorreag@uc.cl momento terapia sistémica para control de la enfermedad.

Existen 3 terapias sistémicas usadas regularmente para el tratamiento de la psoriasis, siendo éstas: metotrexato, ciclosporina y acitretin, que están aprobados por la Food and Drug Administration de los Estados Unidos de Norteamérica (FDA) para el manejo de la psoriasis ${ }^{3,4}$, a los que se han agregado en los últimos años las terapias biológicas. Además, se cuenta con la fototerapia, tanto radiación ultravioleta 
B (UVB) como UVB de banda angosta y radiación ultravioleta A asociada a psolarenos (PUVA).

El metotrexato ha sido usado para el tratamiento de la psoriasis desde la década $1960-69^{5}$, y continúa siendo una terapia efectiva en el tratamiento de pacientes con psoriasis moderadas y severas (compromiso de más de $20 \%$ de superficie corporal), incluida la psoriasis eritrodérmica y psoriasis pustular, además de la refractaria a otros tratamientos como la terapia tópica, fototerapia ${ }^{2,4}$, incluso está indicado en niños con psoriasis severa $^{6}$. También es particularmente benéfico en pacientes con artritis psoriática ${ }^{3,7}$.

El metotrexato es un antimetabolito, cuyo principal efecto adverso en el corto plazo es la toxicidad en la médula ósea ${ }^{4,8}$, que, aunque es poco frecuente, puede ser fatal ${ }^{8,9}$, en tanto en el largo plazo, cobra importancia la hepatoxicidad ${ }^{10-12}$, por lo mismo debe ser evitado o administrado con extremada precaución en pacientes con enfermedades hepáticas. La tasa de fibrosis hepática en pacientes psoriáticos tratados con metotrexato se sitúa entre $0 \%$ y 4\%3,13. Según lo reportado por el Committee on the Safety of Medicines del Reino Unido (CSM), de los 164 casos fatales entre 1969 y 2004, 67 fueron asociados a mielosupresión, 30 por complicaciones pulmonares, 8 por complicaciones hepáticas ${ }^{13}$. Existen otros efectos adversos, como muerte fetal, teratogenicidad, enfermedad pulmonar, reacciones cutáneas, infecciones oportunistas severas, desórdenes gastrointestinales ${ }^{2}$.
El metotrexato tiene una excreción renal, por lo tanto se deberá cuidar su toxicidad, especialmente en pacientes con falla renal y en ancianos; además, se debe evitar asociar con otras drogas que pueden aumentar su toxicidad: antiinflamatorios no esteroidales, ácido acetilsalicílico, sulfas ${ }^{4}$.

Roenigk et al ${ }^{14-18}$ han desarrollado guías clínicas para la monitorización de pacientes en tratamiento con metotrexato (Tabla 1), éstas incluyen la monitorización frecuente de las pruebas hepáticas $\mathrm{y}$, aunque se especifica que no son un buen marcador de daño hepático crónico, tendrían utilidad para detectar daño agudo que aunque es infrecuente, podría tener serias consecuencias en un grupo pequeño de pacientes, se sugiere además, que en pacientes con pruebas hepáticas normales, sin historia de enfermedad hepática ni alcoholismo, no es necesario realizar biopsia hepática hasta alcanzar dosis acumulada de 1 a 1,5 g, repitiendo la biopsia por cada 1 a 1,5 g, si las pruebas hepáticas y biopsias previas son normales.

\section{Material y MÉtodo}

Se diseñó un estudio retrospectivo que tenía como objetivo identificar alteraciones de las pruebas hepáticas que hubieran sugerido toxicidad hepática aguda o crónica y que hubieran conducido a modificación de la conducta terapéutica o un

Tabla 1. G uías para monitorización de pacientes psoriáticos tratados con metotrexato 25,30

\section{Monitorización basal}

- Historia y examen físico completo

- Hemograma completo y recuento de plaquetas

- Pruebas hepáticas (GPT, GOT, FA, bilirrubina, albúmina), niveles creatinina, niveles BUN

- Test VIH (si existe riesgo)

Monitorización del seguimiento

- Hemograma completo y recuento plaquetas semanalmente por 2 semanas, luego mensual

- Pruebas hepáticas mensualmente

- Creatinina, BUN cada 3 ó 4 meses

- Repetir exámenes, después de 7 días que se aumenta la dosis

Dosis

- Dosis de prueba: 2,5-5 mg

- Dosis promedio: $10-15 \mathrm{mg} / \mathrm{semanal}$

- Dosis máxima: $30 \mathrm{mg} / \mathrm{semanal}$

- Mejoría, disminuir cada 2,5 mg/mensuales 
estudio más exhaustivo. Consistió en la revisión de fichas clínicas de pacientes psoriáticos tratados con metotrexato entre los años 2000 y 2005 y que fueron atendidos en el Servicio de Dermatología del Hospital Dr. Sótero del Río y en la consulta privada de uno de los investigadores. El criterio de inclusión fue pacientes con diagnóstico de psoriasis que habían recibido metotrexato como tratamiento, mínimo $7,5 \mathrm{mg}$ a la semana, vía oral, por al menos 4 semanas.

Todos los pacientes fueron instruidos para que evitaran completamente la ingesta de alcohol y, a continuación se les indicó el medicamento una vez a la semana, después de una dosis de prueba de $5 \mathrm{mg}$ seguida por un hemograma completo a la semana. Si no se detectaban alteraciones hematológicas, se indicaba metotrexato $7,5 \mathrm{mg} / \mathrm{semana}$ en dosis única. Se controlaba hemograma y pruebas hepáticas una vez al mes y prueba de función renal cada 3 a 4 meses.

Si no había una alteración significativa de estos exámenes, se aumentaba la dosis $2,5 \mathrm{mg}$ cada mes, hasta un máximo de $25 \mathrm{mg}$ semanales. Los pacientes que alcanzaban una dosis acumulativa de 1,5 g eran derivados para biopsia hepática.

Se consideró como rango normal para cada prueba las cifras comunicadas por cada laboratorio. Sin embargo, para modificar o suspender el tratamiento, se requirió que en el caso de la bilirrubinemia ésta fuera superior a $2 \mathrm{mg} / \mathrm{dl}$, y en el caso de las enzimas hepáticas y fosfatasas alcalinas, el resultado fuera al menos el doble del límite superior del rango normal, informado por el laboratorio de origen.

\section{RESULTADOS}

Se encontró 63 pacientes que cumplían los requisitos mencionados y que acudieron regularmente a sus controles hasta ser dados de alta. Del total, 20 pacientes (32\%) presentaron alguna alteración de sus pruebas hepáticas, la mayoría de sexo masculino, sin embargo, en sólo 9 pacientes (15\%) la alteración superó los $2 \mathrm{mg} / \mathrm{dl}$ en el caso de la bilirrubinemia; 02 veces el límite superior del rango normal, en el caso de las transaminasas y fosfatasas alcalinas (Tabla 2) (Figura 1). No se produjo ninguna falla hepática aguda, ni mielotoxicidad en los pacientes estudiados. La dosis acumulada promedio fue de $576 \mathrm{mg}$ de metotrexato.

Bilirrubina. Dos pacientes (3,2\%) presentaron elevación de la bilimubinemia por sobre los $2 \mathrm{mg} / \mathrm{dl}$, en ambos ocurrió cuando recibían dosis de $10 \mathrm{mg} / \mathrm{sem}$; en la semana 8 y 85, con dosis acumuladas de 70 y $1.275 \mathrm{mg}$, respectivamente. Estos valores comenzaron a bajar al reducir las dosis y no se acompañaron de elevación de las enzimas hepáticas.

\section{Tabla 2. Valores máximos alcanzados, en relación a dosis acumulada, en los pacientes con alteraciones significativas de pruebas hepáticas}

\begin{tabular}{|lccccccc|}
\hline & Sexo & $\begin{array}{c}\text { BT } \\
\mathrm{mg} / \mathrm{dl}\end{array}$ & $\begin{array}{c}\text { GOT } \\
\mathrm{U} / \mathrm{l}\end{array}$ & $\begin{array}{c}\text { GPT } \\
\mathrm{U} / \mathrm{l}\end{array}$ & $\begin{array}{c}\text { GGT } \\
\mathrm{U} / \mathrm{l}\end{array}$ & $\begin{array}{c}\text { FA } \\
\mathrm{U} / \mathrm{l}\end{array}$ & $\begin{array}{r}\text { Dosis acumulada } \\
\mathrm{mg}\end{array}$ \\
\hline Paciente 1 & $\mathrm{M}$ & $2,07^{*}$ & - & - & - & - & 1.275 \\
Paciente 3 & $\mathrm{M}$ & - & $233^{* * S}$ & $321^{* * \mathrm{~S}}$ & - & - & 315 \\
Paciente 7 & $\mathrm{M}$ & $2,25^{*}$ & - & - & - & - & 70 \\
Paciente 11 & $\mathrm{M}$ & - & - & $185^{* *}$ & - & - & 553 \\
Paciente 12 & $\mathrm{M}$ & - & - & $109^{* *}$ & - & - & 1.194 \\
Paciente 13 & $\mathrm{F}$ & - & $70^{* *}$ & - & - & - & 130 \\
Paciente 15 & $\mathrm{M}$ & - & $99^{* *}$ & $97^{* *}$ & - & - & 100 \\
Paciente 17 & $\mathrm{M}$ & - & $103^{* *}$ & $224^{* *}$ & - & - & 45 \\
Paciente 20 & $\mathrm{M}$ & - & 59 & $110^{* *}$ & - & - & 175 \\
\hline
\end{tabular}

BT: bilimubinemia total, GOT: aspartato amino transferasa, GPT: alanino amino transferasa, GGT: gammaglutamiltransferasa, FA: fosfatasas alcalinas.

*Elevación más de $2 \mathrm{mg} / \mathrm{dl}$. **Elevación sobre el doble límite superior. SSuspensión de tratamiento. 


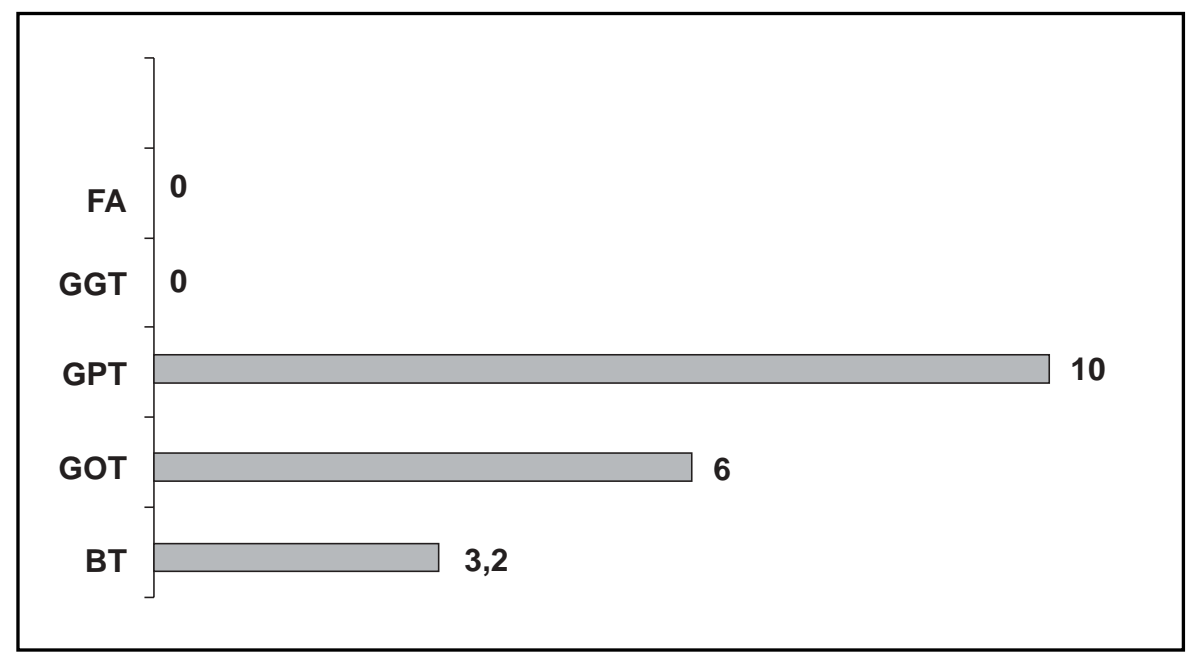

Figura 1. Porcentaje de pacientes con alteración significativa de pruebas hepáticas.

Aspartato amino transferasa (GOT). Cuatro pacientes $(6 \%)$ presentaron elevación de la GOT más allá del doble del límite superior del rango normal. En uno de ellos, la elevación fue 6 veces este límite y además se elevó la GPT, ocumió en la semana 25, con una dosis acumulada de $315 \mathrm{mg}$, lo que motivó la suspensión del tratamiento.

En los otros 3 pacientes, la elevación alcanzó sólo 2 veces el límite superior y se normalizó al disminuir las dosis. Su detección ocurrió en la semana 6, 9 y 13; con dosis acumulativas de 45, 130 y $137 \mathrm{mg}$, respectivamente.

Alanino amino transferasa (GPT). Seis pacientes (10\%) presentaron una anormalidad significativa de GPT, un paciente presentó elevación de 8 veces el margen superior del rango normal y es el mismo que tuvo una fuerte alza de la GOT, lo que obligó a la suspensión del tratamiento. Los controles posteriores evidenciaron una rápida normalización de sus pruebas hepáticas y después de un par de años, el reumatólogo reinstaló el tratamiento por artritis psoriática, sin que haya presentado alteraciones hepáticas en un seguimiento de más de dos años, aunque no se ha alcanzado la dosis que estaba recibiendo al momento de presentar las alteraciones en el primer tratamiento, esto es $17,5 \mathrm{mg}$ a la semana.

Otros 2 pacientes tuvieron un alza de 4 y 5 veces el límite superior del rango normal, en uno de ellos se suspendió el tratamiento, luego de lo cual recuperó rápidamente los valores normales, el otro también presentó alteración significativa de la GOT, todos los valores se recuperaron al bajar la dosis.

Otros 3 pacientes presentaron alteraciones que no iban más allá de 3 veces el límite superior del rango normal y se normalizaron al bajar las dosis.

Estas alteraciones se detectaron en semanas distintas para cada paciente, entre la semana $6 \mathrm{y}$ 84; y con dosis acumulativas también diferentes que van de $45 \mathrm{mg}$ a $1.194 \mathrm{mg}$.

Gamma glutamil transferasa (GGT). En 37 pacientes (59\%), su seguimiento con pruebas hepáticas incluyó además GGT, en ninguno se observó un alza superior a dos veces el límite superior del rango normal.

Fosfatasas alcalinas. No se detectó elevación significativa en ningún paciente.

Aunque se buscó, no se encontró una relación directa, es decir, a mayor dosis de metotrexato mayor grado de alteración, entre las dosis semanales del medicamento y la anormalidad de los exámenes. En general estas alteraciones se detectaron con dosis que fueron entre 7,5 y 22,5 mg de metotrexato a la semana; tampoco existió relación directa entre dosis acumulada y riesgo de alteración de prueba hepáticas, las alteraciones se detectaron con dosis acumuladas entre 45 y 1.274 mg. 
Biopsia hepática. Sólo un paciente sobrepasó 1,5 g como dosis acumulativa, se le realizó biopsia hepática que fue informada como normal, sin signos de fibrosis hepática.

\section{CONCLUSIONES}

De los resultados obtenidos en nuestro estudio, llama la atención que en un número importante de nuestros pacientes psoriáticos, un tercio, presenta alguna alteración de sus pruebas hepáticas a lo largo del tratamiento con metotrexato, aunque sólo en la mitad de ellos esta alteración es significativa, sin embargo todos se normalizan al suspender o bajar las dosis, lo que habla de un impacto mínimo a nivel del parénquima hepático ${ }^{2,11,19}$ o de una alta sensibilidad de los exámenes para daño agudo, lo que justificaría plenamente el empleo regular de las pruebas hepáticas en el seguimiento de los pacientes con psoriasis tratados con metotrexato. Además, se debe controlar seriadamente hemograma (mensualmente hasta estabilizar dosis de metotrexato, luego cada 2 ó 3 meses) ${ }^{14,18}$ y función renal ${ }^{2,9}$.

También es significativo constatar que aparentemente no hay relación con la dosis semanal y la dosis acumulativa, lo que hace pensar en un fenómeno idiosincrático e impredecible, por lo que el monitoreo debería ser, entonces, en cualquier fase del tratamiento; lo cual estaría de acuerdo a lo publicado $2,14,20$

Se ha reportado que el riesgo relativo de aumento de transferasas en hombres psoriáticos tratados con metotrexato es de 2,7 (CI 95\%, 0,7$10,7)$, para pacientes mayores de 40 años es de 1,7 (CI 95\%, 0,6-4,9), y en ausencia de esteatohepatitis previa es de 0,8 (CI 95\%, 0,3-2,8) ${ }^{21}$.

Sólo uno de nuestros pacientes alcanzó una dosis suficientemente alta $(1,5 \mathrm{~g})$ que obligara a realizar biopsia hepática, por lo que no se puede evaluar ni concluir cuál es el rol que el monitoreo de las pruebas hepáticas tiene en pacientes con dosis acumulativas elevadas.

De la literatura sabemos que hay un bajo riesgo de desarrollar daño hepático significativo en pacientes que reciben metotrexato en bajas dosis semanales $9,22,23$. Zachariae et al analizaron 700 biopsias hepáticas de pacientes psoriáticos tratados con metotrexato, 39 tomaron metotrexato por más de 5 años, de ellos $25 \%$ desarrolló cirrosis hepática; la primera detectada a los 2,2 g de dosis acumulada ${ }^{24}$. Algunos pacientes siguieron tomando metotrexato, a pesar de los resultados en la biopsia, pero al repetir la biopsia no se encontró progresión de la fibrosis, sugiriendo que la cirrosis inducida por metotrexato no sería progresiva ${ }^{11,25}$, incluso reversible. Psoriasis Task Force of the American Academy of Dermatology recomienda realizar biopsia hepática en pacientes con psoriasis después que el tratamiento se ha estabilizado, y después de alcanzar la dosis acumulada de $1,5 \mathrm{~g}$ de metotrexato, en la práctica cada 18 meses a 2 años por paciente ${ }^{4,9,23}$. Es importante considerar que el riesgo de daño por biopsia hepática es de $1 \%$ a $2 \%$, y riesgo de mortalidad es de $0,01 \%$ a $0,1 \% 26$.

Prácticamente todas las pautas de manejo de metotrexato en pacientes psoriáticos incluyen la recomendación de realizar periódicamente pruebas hepáticas (Tabla 1), aun cuando se destaca que no son sensibles para detectar la fibrosis hepática asociada al empleo de metotrexato en forma crónica ${ }^{2,13}$, sí servirían para detectar daño hepático agudo por metotrexato; sin embargo la literatura es pobre en lo que se refiere a este daño agudo y su detección.

Dado que éste fue un estudio retrospectivo, no se investigó la asociación entre la alteración de pruebas hepáticas por metotrexato y el consumo de otros medicamentos (AINES, sulfas, fenitoína, antibióticos) ${ }^{2}$ o alcohol, que podrían aumentar la toxicidad del metotrexato $7,10,22,27 \mathrm{y}$, por lo tanto, alterar las pruebas hepáticas, y que podnáan explicar alguna de las alteraciones menores encontradas. Existe un marcador sanguíneo asociado a fibrosis hepática llamado el péptido amino-terminal de procolágeno tipo III (PIIIP), pero en Chile no está disponible. En Europa, $20 \%$ de los dermatólogos lo usa rutinariamente para la detección de fibrosis hepática secundaria a metotrexato ${ }^{9}$, lo que lleva a que la monitorización con pruebas hepáticas se realice más espaciada, esto es cada 2 meses o más. Se recomienda controlar niveles de PIIIP cada 3 meses durante un tratamiento continuo de metotrexato ${ }^{28,29}$, con indicación de biopsia, sólo en caso de niveles persistentemente altos ${ }^{21}$. Esto ha logrado disminuir 7 veces la necesidad de biopsia hepática en paciente psoriáticos tratados con metotrexato, con un costo menor y la preferencia de los pacientes $^{26}$. Por último, es importante que todos los 
pacientes que reciben metotrexato, estén familiarizados con los posibles riesgos de la droga, y sepan claramente cómo administranla (dosis únicas semanales). Debe conocer y evitar el consumo de sustancias que interactúen con éste y sus senias consecuencias.

En resumen, no se detectó un patrón específico de comportamiento de las pruebas hepáticas en

\section{REFERENCIAS}

1. Valdés MP, Schroeder F, Roizen G, Honeyman J, SÁnchez L. Eficacia y seguimiento en el largo plazo de pacientes con psoriasis vulgar moderada a severa en tratamiento con infliximab (Remicade $\left.^{\circledR}\right)$. Rev Méd Chile 2006; 134: 326-31.

2. NaLdi L, Grifriths CEM. Traditional therapies in the management of moderate to severe chronic plaque psoriasis: an assessment of the benefits and risks. Br J Dermatol 2005; 152: 597-615.

3. LeBworl M, AL S. Treatment of psoriasis. Part 2. Systemic therapies. J Am Acad Dermatol 2001; 45: 649-61.

4. Griffiths CEM, Clark CM, Chalmers RJG, L Wan Po A, Wiшuams HC. A systematic review of treatments for severe psoriasis. Health Technol Assess 2000; 4: 1-125. Search date 1999; primary sources Medline, Embase, and Cochrane Register of RCTs.

5. WARREN RB, GRIFFTHS CME. The potential of pharmacogenetics in optimizing the use of methotrexate for psoriasis. Br J Dermatol 2005; 153: 869-73.

6. Dogra S, Handa S, Kanwar AJ. Methotrexate in severe childhood psoriasis. Pediatric Dermatology 2004; 21: 283-4.

7. Whiting-O'Keefe QE, Fye KH, Sack KD. Methotrexate and histologic hepatic abnormalities: a meta-analysis. Am J Med 1991; 90: 711-16.

8. Solganick J, Tan MH, LeBwohl M. Systemic treatments: adverse effects of methotrexate. Psoriasis Forum 1997; 3: 5.

9. BoFfA MJ. Methotrexate for psoriasis: current European practice. A postal survey. J Eur Acad Dermatol Venerol 2004; 104: 196-202.

10. WeST SG. Methotrexate hepatotoxicity. Rheum Dis Clin North Am 1997; 23: 883-915.

11. Zachariae $H$, Sogaard H, HeickendorfF L Methotrexate-induced liver cirrhosis. Clinical, histologi$\mathrm{cal}$ and serological studies - a further 10 year follow-up. Dermatology 1996; 192: 343-6. pacientes psoniáticos tratados con metotrexato, ya que su alteración seńa dosis independiente, sin embargo, permitinan detectar precozmente algún daño hepático agudo. El que estos parámetros se normalicen con facilidad, induce a pensar que reflejan un daño mínimo, por lo tanto son suficientemente sensibles y justificarían su monitoreo permanente.

12. Contreras J, Poniachik J, Planzer M, Lazarte R, SMok G, O KSENBERg D et al. [Drug induced liver disease: clinical and pathological patterns in 33 cases]. Rev Méd Chile 2003; 131: 1128-34.

13. MacDonald A, BuRden AD. Noninvasive monitoring for methotrexate hepatotoxicity. Br J Dermatol 2005; 152: 405-8.

14. Roenigk HH JR, Auerbach R, Maibach H, Weinstein G, LeBwoнL M. Methotrexate in psoriasis: consensus conference. J Am Acad Dermatol 1998; 38: 478-85.

15. Roenigk HH, Maibach HI, Weinstein GD. Guidelines on methotrexate therapy for psoriasis. Arch Dermatol 1972; 105: 363-5.

16. Roenigk HH, Maibach HI, Weinstein GP. Methotrexate therapy for psoriasis: guideline revisions. Arch Dermatol 1973; 108: 35.

17. Roenigk HH, Auerbach R, Maibach HI, Weinstein GD. Methotrexate guidelines - revised. J Am Acad Dermatol 1982; 6: 145-55.

18. Roenigk HH, Auerbach R, Maibach HI, Weinstein GD. Methotrexate in psoriasis: revised guidelines. J Am Acad Dermatol 1988; 19: 145-56.

19. Heydendael VMR, Spuls PI, Opmeer B, M De Borgie C, Reitsma J, Goldschmidt W et al. Methotrexate versus cyclosporine in moderate to severe chronic plaque psoriasis. N Engl J Med 2003; 349: 65865.

20. Berends MA, Snoek J, De Jong EM, Van De Kerkhof PC, Van OiJen MG, Van Krieken JH et al. Liver injury in long-term methotrexate treatment in psoriasis is relatively infrequent. Aliment Pharmacol Ther 2006; 24: 805-11.

21. Heydendael VMR, Spuls P, Bossuyt P, Bos J, A De Re M. Analysis of risk factors in psoriatic patient with methotrexate - induced increases in transaminase levels. Arch Dermatol 2004; 104: 1289.

22. Lanse SB, Arnold GL, Gowans JD, Kaplan MM. Low incidence of hepatotoxicity associated with longterm, low-dose oral methotrexate in treatment of 
refractory psoriasis, psoriatic arthritis, and rheumatoid arthritis. An acceptable risk/benefit ratio. Dig Dis Sci 1985; 30: 104-9.

23. Aithal GP, Haugk B, Das S, Card T, Burt AD, RECORD CO. Monitoring methotrexate-induced hepatic fibrosis in patients with psoriasis: are serial liver biopsies justified? Aliment Pharmatol Ther 2004; 19: 391-9.

24. Zachariae H, Kragbale K, Sogaard H. Methotrexate induced liver cirrhosis. Studies including serial liver biopsies during continued treatment. $\mathrm{Br} \mathrm{J}$ Dermatol 1980; 102: 407-12.

25. Zachariae $H$, Sogaard $H$. Methotrexate -induced liver cirrhosis. A follow-up. Dermatologica 1987; 175: 178-82.

26. Chalmers RJG, Kirby B, Smith A, Burrows P, Little R, Horan M et al. Replacement of routine liver biopsy by procollagen III aminopeptide for monitoring patients with psoriasis receiving long-term methotrexate: a multicentre audit and health economics analysis. Br J Dermatol 2005; 154: 444-50.

27. Shergy WJ, Polisson RP, Caldwell DS, Rice JR, PISETSKY DS, ALEN NB. Methotrexate-associated hepatotoxicity: retrospective analysis of 210 patients with rheumatoid arthritis. Am J Med 1988; 85: 771-4.

28. Boffa MJ, Smith A, Chalmers RJ, Mitchell DM, ROWAN B, WARNES TW ET AL. Serum type III procollagen aminopeptide for assessing liver damage in methotrexate-treated psoriatic patient. $\mathrm{Br}$ J Dermatol 1996; 135: 538-44.

29. Zachariae H, HeickendorfF L, Sogaard $H$. The value of aminoterminal propeptide of type III procollagen in rutine screening for methotrexato induced liver fibrosis: a 10 years follow-up. Br J Dermatol 2001; 144: 100-3.

30. SAPORITO F, MENTER MA. Methotrexate and psoriasis in the era of the new biologic agents. J Am Acad Dermatol 2004; 50: 301-10. 